ARTIGO DE PESQUISA - RESEARCH ARTICLE

\title{
DIVERSIDADE DE PLANTAS PARA HIPERTENSÃO, HIPERCOLESTEROLEMIA E PROBLEMAS RENAIS USADAS POR UMA COMUNIDADE RURAL NA SERRA DA MANTIQUEIRA PAULISTA
}

\author{
PLANT DIVERSITY FOR HYPERTENSION, HYPERCHOLESTEROLEMIA AND RENAL \\ PROBLEMS USED BY A RURAL COMMUNITY IN SERRA DA MANTIQUEIRA \\ PAULISTA
}

Livia Cristina Pereira BARROS ${ }^{1}$; Lin Chau MING $^{1 *}$; Sandra Maria Pereira da SILVA²; Roseli Buzanelli TORRES ${ }^{3}$

1- Departamento de Horticultura, Faculdade de Ciências Agronômicas, Universidade Estadual Paulista, Campus de Botucatu - SP, liviaflorestaviva@hotmail.com; autor para correspondência *lin.ming@unesp.br; 2 -Pólo Regional de Desenvolvimento Tecnológico dos Agronegócios do Vale do Paraíba, Pindamonhangaba - SP, sandrasilva@aptaregional.sp.gov.br; 3- Instituto Agronômico de Campina, Campinas/SP; rbtorres@ iac.sp.gov.br

\section{RESUMO}

Submitted: 04/11/2019; Accepted: 27/01/2019

Esta pesquisa teve como objetivo principal realizar levantamento etnobotânico junto à comunidade rural do Bairro dos Marins, município de Piquete, São Paulo, sobre o conhecimento de plantas medicinais com atividades de controle da pressão arterial, colesterol e problemas renais. O estudo objetivou também verificar como os moradores reconhecem e nomeiam as doenças estudadas, além de identificar quais espécies medicinais são conhecidas e usadas pelos moradores, sua forma de utilização e preparo. A coleta de dados foi baseada em entrevistas semiestruturadas e estruturadas, por meio das técnicas de observação participante e de "bola-de-neve"; esta última consistiu em conversar com algumas pessoas da comunidade e perguntar se conheciam moradores que tinham significativo conhecimento sobre plantas medicinais e com esta técnica foram indicadas 20 pessoas. Foram coletados dados socioculturais, levantados os problemas de saúde mais recorrentes na comunidade e as espécies usadas para tratar cada um deles, bem como as pessoas conhecem as plantas para os sintomas das doenças. Foram citadas 57 espécies medicinais para as doenças estudadas, distribuídas em 33 famílias botânicas. Com a pesquisa, apesar de ter se restringido a alguns grupos de doenças, tornou-se possível verificar que no Bairro dos Marins existe intenso uso de plantas medicinais nos cuidados primários à saúde, para os grupos das doenças estudadas.

Palavras-chave: etnobotânica, espécies medicinais, doenças, Antropologia, biodiversidade.

\begin{abstract}
The main objective of this research was to do an ethnobotanical survey in the rural community of Bairro dos Marins, Piquete municipality (São Paulo State), on their knowledge about medicinal plants with activity on blood pressure, cholesterol control, and kidney problems. Other objectives are to verify how the inhabitants know and name the studied diseases, to identify which species of medicinal plants are known and used by the inhabitants, how they prepare and use each one. The collection of data was based on methods used in Anthropology, like semi-structured and structured interviews and also participant observation. It was used the "snow ball" technique, which consists on talking to some people of the community and asking them whether they know some inhabitants who have a significant knowledge about medicinal plants. Sociocultural data were collected, as well as the community's most common health problems and the plants used to treat each problem. Twenty persons were indicated with the use of this technique. Fifty seven medicinal plants were found for the studied diseases distributed in thirty three botanical families. As conclusion, it was possible to verify that the community Bairro dos Marins widely uses medicinal plants to treat primary health problems, for the studied diseases group.
\end{abstract}

Keywords: ethnobotany, medicinal species, diseases, Anthropology, biodiversity.

\section{INTRODUÇÃO}

O Brasil inclui-se entre os países de maior biodiversidade mundial, com espécies distribuídas em diversos biomas. Entre estes biomas está a Mata Atlântica, com mais de 20 mil espécies vegetais, situada entre os 25 "hotspots" mais importantes do mundo (MYERS et al., 2000). Os poucos maciços 
florestais que restam da Mata Atlântica no Estado de São Paulo encontram-se distribuídos em locais de relevo acidentado, como a região da Serra do Mar, Vale do Ribeira e a Serra da Mantiqueira, o que dificulta tanto o extrativismo quanto a agricultura em larga escala, levando à conservação destes ambientes mesmo depois de seguidos ciclos de exploração e expansão agrícola, sofridas por este bioma.

Neste contexto, existem populações rurais que convivem com este ambiente há séculos e detêm um rico conhecimento sobre os recursos naturais da Floresta Atlântica. Com a intensa migração para maiores centros urbanos, que se observa no Brasil ao longo do processo de industrialização e atualmente, verifica-se que este conhecimento tradicional tende a se perder em decorrência da erosão cultural. Este conhecimento não está documentado em livros e enciclopédias, mas guardado com as pessoas, as quais, em sua maioria, transmitem estas preciosas informações de forma oral.

$\mathrm{Na}$ Serrada Mantiqueira paulista esta realidade de erosão cultural vem associada à escassez de estudos botânicos, tanto qualitativos quanto quantitativos, sobre espécies de plantas com potencial medicinal, principalmente quando se fala de estudos etnobotânicos. Sendo assim, a presente pesquisa parte de informações coletadas junto aos moradores do Bairro dos Marins, que faz parte da APA da Serra da Mantiqueira, e tem como objetivo levantar o conhecimento associado ao uso de plantas medicinais para doenças como hipertensão arterial, hipercolesterolemia e problemas ligados aos rins e bexiga, doenças de grande prevalência na comunidade estudada e também na população brasileira em geral.

\section{MATERIAL E MÉTODOS \\ Local de estudo}

O município de Piquete, que possui uma população de 14.475 habitantes, localiza-se no Estado de São Paulo na região do Vale do Paraíba, encravada aos pés da Serra da Mantiqueira. Está a $200 \mathrm{~km}$ da capital do Estado (Figura 1) e tem as seguintes coordenadas geográficas: $22^{\circ} 33$ '45" de Latitude Sul e 45 $11^{\prime}$ ' 15" de Longitude Oeste. A comunidade estudada é conhecida como Bairro dos Marins e se formou com a chegada de mineiros e tropeiros em 1937. O núcleo do bairro está a uma altitude de 800 $\mathrm{m}$, chegando a $2.420 \mathrm{~m}$ no Pico dos Marins.

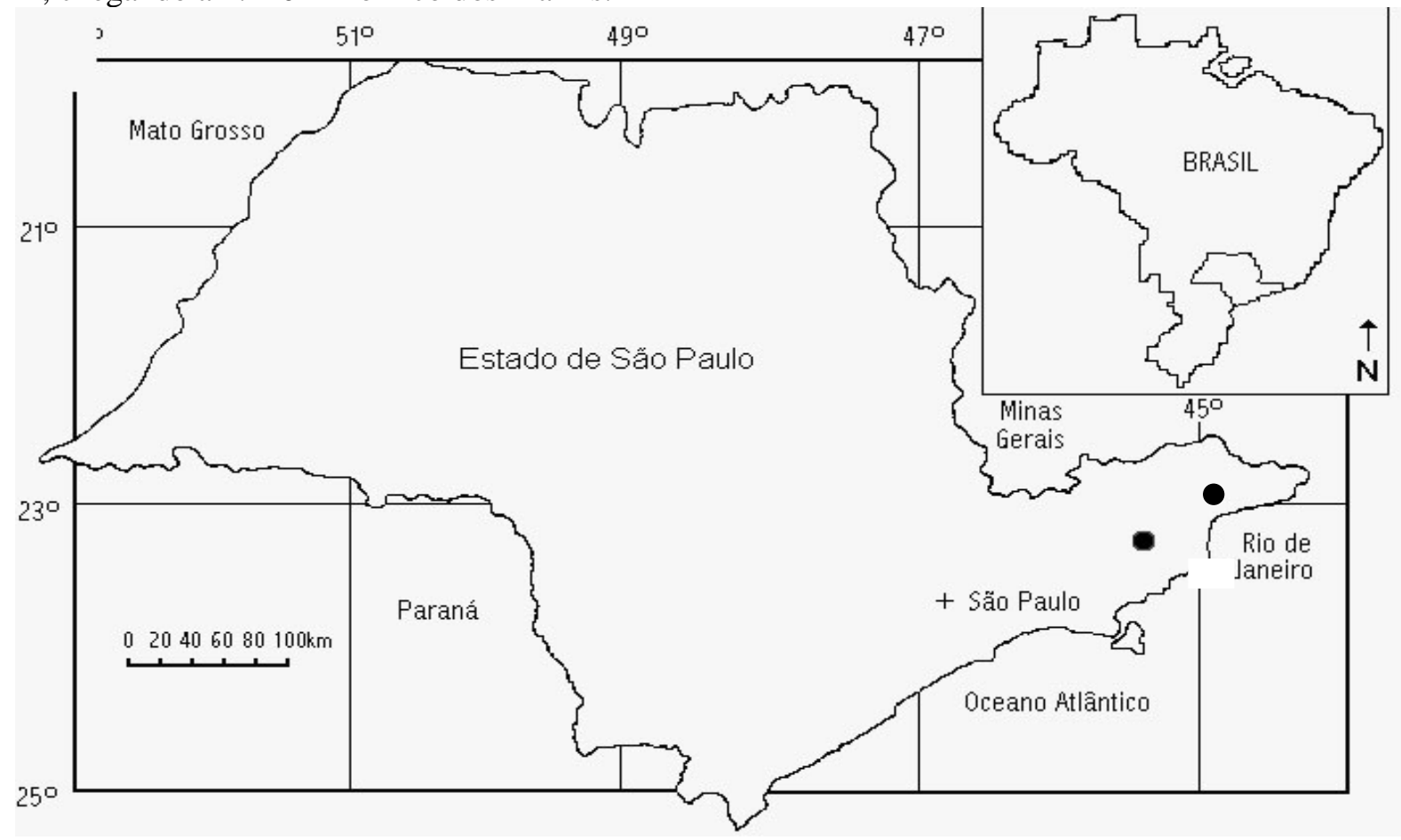

Figura 1. Localização do município de Piquete, São Paulo (adaptado SMA, 1998).

As entrevistas foram divididas em três etapas para viabilizar a estruturação da pesquisa e escolher as doenças a serem estudadas. Na primeira etapa, foram feitas visitas a todas as casas do Bairro dos Marins. Foram entrevistadas 74 pessoas, mediante entrevistas estruturadas e semiestruturadas. A segunda etapa consistiu na coleta de dados socioculturais e foram levantados os problemas de saúde mais recorrentes na comunidade e as plantas usadas para tratar cada um deles. Para a terceira etapa foi usada a técnica de amostragem "bola de neve" (MARTIN, 1995; ALEXIADES, 1996), que consistiu 
em conversar com algumas pessoas da comunidade e perguntar se conheciam moradores que tinham significativo conhecimento sobre plantas medicinais; com esta técnica foram indicadas 20 pessoas. Com este grupo de entrevistados optou-se por utilizar entrevistas semiestruturadas com perguntas abertas a fim de verificar como esses especialistas nomeiam as afecções estudadas e como reconhecem os problemas de saúde a partir dos sintomas, tendo também sido utilizada a metodologia da observação participante durante o período de permanência na comunidade. Neste artigo se encontram os resultados da terceira etapa desta pesquisa.

Com todos os entrevistados foram obtidos os termos de consentimento livre e esclarecido (TCLE) e o projeto obteve autorização do Comitê de Ética da UNESP - Botucatu, SP, não tendo sido encaminhado solicitação ao CGEN-Ministério do Meio Ambiente.

As plantas citadas foram coletadas no ambiente onde elas crescem com ajuda e presença dos entrevistados. As espécies foram prensadas e identificadas por especialistas botânicos de algumas instituições de pesquisa e universidades. O sistema de classificação adotado para famílias foi o APG IV (APG IV, 2016) e a nomenclatura botânica foi atualizada pela Lista de Espécies da Flora do Brasil 2020, em construção (FLORA DO BRASIL, 2019).

As plantas coletadas foram depositadas no Herbário do Jardim Botânico do Instituto Agronômico de Campinas com duplicatas enviadas ao Herbário Irina Delanova Gemtchújnicov UNESP - Botucatu, SP.

\section{RESULTADOS E DISCUSSÃO \\ Dados socioeconômicos}

Dentro do grupo de 20 especialistas entrevistados, $25 \%$ são do gênero masculino e $75 \%$ do gênero feminino (Figura 1). A menor idade encontrada foi 36 e a maior 76 anos. A faixa etária de maior número de entrevistados ficou entre 61 e 70 anos, aparecendo uma alta proporção de idosos, pois $60 \%$ dos entrevistados possuíam mais de 60 anos de idade. Quanto à origem destes moradores, verificou-se que $45 \%$ dos entrevistados nasceram e viveram na comunidade durante toda a vida e apenas um dos entrevistados não nasceu na região.

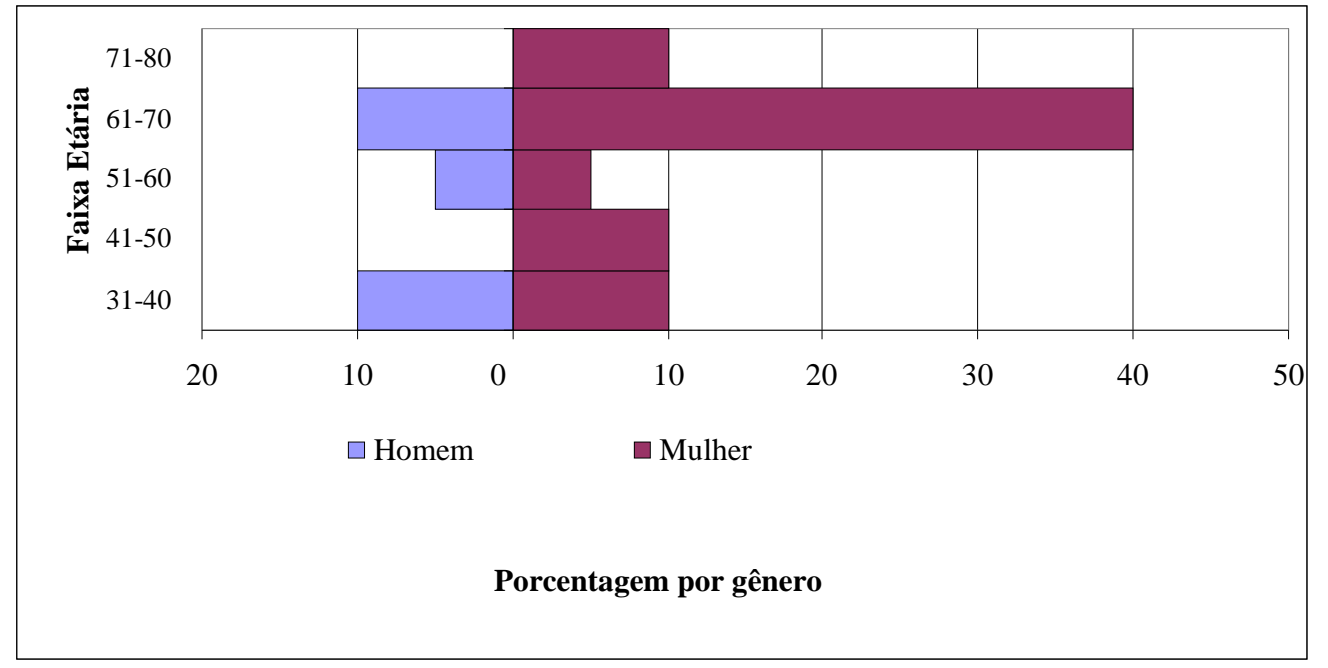

Figura 2: Distribuição dos especialistas entrevistados por estrutura etária e gênero, no Bairro dos Marins, Piquete- SP.

Quanto ao grau de escolaridade, $90 \%$ dos entrevistados não terminaram o ensino fundamental. Essa realidade pode ser explicada, pois a escola existente no bairro foi construída em 1972, a partir da doação do terreno por um dos moradores. Anteriormente a este fato, as aulas eram dadas no barracão da capela. Atualmente, a escola ensina até a $4^{\mathrm{a}}$ série do ensino fundamental. Para continuar os estudos, as crianças se deslocam até Piquete onde existem escolas que oferecem até o ensino médio.

A ocupação dos entrevistados varia de acordo com o gênero, pois na comunidade as tarefas são bem divididas: $50 \%$ fabricam e vendem queijo nos dias de hoje e $85 \%$ já tiveram o queijo como principal renda da família. Entre os entrevistados do gênero masculino, dois criam rebanho bovino para a fabricação do queijo e fazem o transporte e venda de animais de pequeno porte, como galináceos e suínos. Um dos entrevistados é dono da única mercearia do Bairro dos Marins; outro é artesão, serrador e mateiro aposentado, enquanto outro trabalha tirando leite e como caseiro para outras pessoas. A 
ocupação das mulheres entrevistadas varia, sendo $25 \%$ artesãs, $53 \%$ queijeiras e $35 \%$ donas-de-casa aposentadas. Essas categorias podem sobrepor-se, existindo mulheres que, mesmo aposentadas, fazem queijo e artesanato para complementar a renda da casa.

As formas de aprendizagem do conhecimento sobre as plantas medicinais são predominantemente de forma oral e dentro da própria comunidade, onde $45 \%$ aprenderam a usar e conhecer as plantas apenas ouvindo a explicação de outras pessoas; $30 \%$ aprenderam tanto ouvindo a explicação quanto observando outras pessoas fazendo; $25 \%$ afirmaram que durante sua experiência de aprendizado ajudaram quem os ensinavam tanto na coleta de plantas, quanto na preparação de remédios caseiros à base de ervas medicinais. Nenhum que respondeu a entrevista disse ter aprendido sobre as plantas medicinais por conta própria.

\section{Percepção das doenças estudadas segundo os entrevistados}

O sistema classificatório popular não se encontra ordenado e disponível sob o domínio de algum usuário ou praticante. $\mathrm{Na}$ verdade, ele se encontra disperso por uma infinidade de usuários que se apropriam apenas de fragmentos dos sistemas. Não se constitui, portanto, um sistema no sentido de ter sido elaborado, formalizado intelectualmente, como encontrado na academia, tornando-se então mais ou menos coerente, obedecendo assim a determinadas regras de ordenação (CARRARA, 1995).

As noções, como as de saúde e doença, dentro de sociedades e a percepção dos indivíduos que compõem estas sociedades sobre esses conceitos, referem-se a fenômenos complexos que conjugam fatores biológicos, sociológicos, econômicos, ambientais e culturais. Nesse sentido, entender o "êmico", o ponto de vista do "outro", consiste numa das principais ferramentas para compreensão de suas atitudes, processo de cura, percepções e o uso dos recursos vegetais para o equilíbrio do corpo e espírito.

Para os entrevistados do Bairro dos Marins os conceitos de saúde/doença estão ligados à ausência ou à presença de dor, e à boa aparência do organismo (físico), isto é, quando o corpo está com uma aparência saudável é sinal de saúde. Quando perguntado aos entrevistados se eles consideravam a pressão alta, o colesterol elevado e problemas que atacam os rins e a bexiga como doenças, todos afirmaram que sim. Entre os entrevistados, registrou-se o relato de doenças que só se sabe que está curado quando se faz exames e o médico garante que a doença melhorou. Esta constatação demonstra que, de alguma maneira, a classificação e o conceito de doenças estão sendo influenciados pela medicina oficial (medicina não tradicional). Para Amorozo (1996), isso não destrói os esquemas explicativos preexistentes, mas acrescenta novas possibilidades a estes.

Neste estudo, procurou-se compreender a visão da comunidade no que diz respeito às doenças propostas. Sendo assim, para cada doença foi estabelecida, segundo os próprios entrevistados, uma listagem dos modos de compreensão das doenças e sintomas estudados, assim como a etimologia de cada uma. Os nomes vernaculares dados às doenças e aos sintomas relacionados às três doenças estudadas foram "traduzidos" pela pesquisadora com auxílio dos profissionais do Programa de Saúde da Família (PSF) que atendem no Bairro dos Marins, e em conversas informais feitas com médicos da área da Urologia, Cardiologia e Homeopatia. Também com o auxílio desses profissionais foi possível realizar a classificação dos sintomas relacionados à pressão alta, descritos pelos entrevistados representados no Quadro 1. Os sintomas descritos não variaram muito, porém dois dos entrevistados que têm a doença disseram não sentir nenhum sintoma.

\section{Pressão alta}

No Bairro dos Marins, a pressão é aferida por uma moradora, que antigamente, quando o acesso aos médicos era mais difícil e não existia o PSF, ajudava as pessoas na aferição da pressão e aplicava injeções. A pressão arterial também pode ser medida mensalmente com as visitas da equipe do PSF. Entre o grupo de 20 entrevistados, a pressão arterial aparece em $20 \%$ deles (sozinha ou associada a outras doenças), porém, todos os entrevistados já ouviram falar da doença e conhecem alguém que tenha. Os nomes dados à hipertensão no Bairro dos Marins, segundo os entrevistados, são: pressão alta, pressão desregulada ou pressão nervosa.

Para os participantes da pesquisa, a pressão alta está relacionada com as emoções e variações de humor diárias, principalmente a emoções ligadas ao Sistema Nervoso Central, como ansiedade, angústia e desespero. 
Barros et al. Diversidade de plantas para hipertensão, hipercolesterolemia e problemas renais usadas por uma comunidade rural na Serra da Mantiqueira Paulista. Ethnoscientia 5, 2020. D.0.I.: 10.22276/ethnoscientia.v5i1.265

Quadro 1- Sintomas descritos pelos especialistas entrevistados para diagnosticar a pressão alta no Bairro dos Marins, Piquete- SP.

\begin{tabular}{|c|c|c|}
\hline Sintomas ligados a dores corporais & Sintomas ligados a emoções & Sintomas ligados a sensações \\
\hline Dor na nuca & Nervoso & Calor intenso \\
\hline Dor de cabeça & Desespero & Coração disparado \\
\hline Dor no peito & Angústia & Vontade de vomitar \\
\hline Dor no braço & Ansiedade & Suor frio \\
& & Visão turva \\
\cline { 3 - 3 } & & Cansaço \\
\cline { 3 - 3 } & & Perna inchada \\
\cline { 3 - 3 } & & \\
\cline { 3 - 3 } & & \\
\end{tabular}

Foram citadas 27 espécies medicinais para o tratamento da hipertensão (Quadro 2), sendo que a mais citada foi Lippia alba (Mill.) N.E. Br (erva-cidreira), com 14 citações e Cymbopogon citratus (DC.) Stapf. (capim-cidreira), com 10 citações. Nos Marins, o capim-cidreira pode ser usado de diversas formas para baixar a pressão sanguínea, sendo a mais comum o chá das folhas frescas ou secas e na forma de suco das folhas frescas. Dentre as plantas citadas para hipertensão, algumas têm o efeito antihipertensivo comprovado cientificamente como Allium sativum L. (alho), Melissa officinalis L. (melissa), Cuphea carthagenensis (Jacq.) J.F.Macbr (sete-sangrias) (TESKE e TRENTINI, 1995) Mentha spicata L. (hortelã-preta) e Cecropia glaviovii Snethl. (embaúva) (MATOS et al., 2001).

\section{Colesterol}

Segundo os entrevistados, o colesterol é uma doença recente, detectada quando o acesso aos exames de sangue ficou mais fácil, pois antigamente não se ouvia falar de colesterol. Em relação à causa da doença, $60 \%$ dos entrevistados disseram que a hipercolesterolemia está ligada a hábitos alimentares irregulares como a ingestão de carne vermelha, leite integral, manteiga, ou seja, todo o tipo de alimento que tenha alto teor de gordura. Dentre o grupo de entrevistados, apenas 5\% apresentam o colesterol elevado.

Os nomes dados a esta doença na comunidade são: gordura nas veias, sangue grosso, limo nas veias, limo nas tripas e sangue gordo. No que diz respeito aos sintomas, $20 \%$ dos entrevistados que apresentam colesterol elevado afirmam não sentir nenhuma dor ou desconforto, enquanto os $5 \%$ dos entrevistados com hipercolesterolemia relatam sentir dores e inchaços nos tornozelos, sensação de peso no corpo, moleza e cansaço.

Na comunidade é comum, para a cura e prevenção da hipercolesterolemia, a citação de plantas que tenham atividade depurativa do sangue. Foram citadas 12 espécies medicinais (Quadro 2) para diminuir os níveis de colesterol no sangue, sendo a mais citada Baccharis conyzoides (Less.) DC. (carqueja; cinco citações), seguida de Solanum melongena L. (berinjela). O número baixo de espécies citadas para problemas ligados ao aumento do colesterol no sangue pode estar relacionado ao fato de ser uma doença assintomática, a não ser em casos mais graves, onde seu diagnostico só pode ser feito por meio de exames de sangue pedidos por médicos.

\section{Dor nos rins e bexiga}

Dentre o grupo de entrevistados, $70 \%$ afirmaram ter ou já ter tido algum problema na bexiga ou nos rins, exatamente por causa dos sintomas ligados a dores intensas nas costas e na hora de urinar, sendo essa doença de fácil diagnóstico mesmo para leigos. No Bairro dos Marins, os principais problemas nos rins e bexiga são cálculos renais e infecções urinárias. Os nomes dados para esses males são: areia nos rins, pedra nos rins, dor descadeiras, dor de urina, dor de bexiga. Os principais sintomas apontados são dor nas costas, dor na barriga, dor na hora de urinar, urina presa, urina fedida, urina com sangue, urina pouquinho e toda hora, ardência no xixi, bexiga presa, bexiga pesada e vômito.

Para problemas nos rins e bexiga, foram citadas 30 espécies, sendo as mais citadas Phyllanthus tenellus Roxb. L. (quebra-pedra-falso) e Costus spicatus (Jacq.) Sw. (cana-do-brejo). Na comunidade, observou-se o costume de associação de algumas plantas no preparo de remédios caseiros para os rins. As plantas geralmente usadas neste preparado são: milho, abacate, quebra-pedra e cana-do-brejo. Observou-se também que esta mistura varia de acordo com a disponibilidade das plantas, e podem ser substituídas por outras também usadas como diuréticas, como quebra-pedra-falso (Phyllanthus tenellus Roxb.) e amora (Morus nigra L.). 


\section{Plantas utilizadas}

Para as três doenças alvo deste estudo, foram citadas 57 espécies (Quadro 2), pertencentes a 33 famílias botânicas, sendo 14 espécies repetidas para pelo menos duas doenças. Diversos fatores podem contribuir para que uma população conheça ou não espécies medicinais. Neste caso, atribui-se um número maior de espécies citadas para doenças que têm os sintomas mais facilmente reconhecidos pela comunidade (problemas nos rins e bexiga). Outro aspecto que pode exercer influência no número de espécies conhecidas para determinada doença é o número de casos da doença entre o grupo estudado. Nota-se que o colesterol teve a menor incidência entre as doenças estudadas, e para o colesterol o número de espécies conhecidas também foi menor. $\mathrm{O}$ fato de a doença estar menos tempo presente na vida dos entrevistados, pode também ter influenciado no número de espécies conhecidas.

Quadro 2- Espécies medicinais, citadas pelos entrevistados do Bairro dos Marins, Piquete- SP, para as doenças alvo deste estudo. Legenda: $\mathrm{HPT}=$ hipertensão, $\mathrm{CLT}=$ colesterol elevado, $\mathrm{PRB}=$ problemas relacionados aos rins e bexiga. Número da exsicata: IAC - Instituto Agronômico de Campinas, I.C.= não coletada.

\begin{tabular}{|c|c|c|c|c|c|c|}
\hline Família & Espécies & Nome Popular & HPT & CLT & PRB & $\mathrm{N}^{0}$ exsicata \\
\hline Alismataceae & $\begin{array}{l}\text { Echinodorus grandiflorus } \\
\text { (Cham. \& Schltr.) Micheli }\end{array}$ & Chapéu-de- couro & & & $\mathrm{X}$ & IAC 48435 \\
\hline Amaranthaceae & Hebanthe paniculata Mart. & Cipó-sumo & & $\mathrm{X}$ & & IAC 48752 \\
\hline Amaryllidaceae & Allium sativum $\mathrm{L}$. & Alho & $\mathrm{X}$ & & & I.C. \\
\hline Annonaceae & Annona muricata L. & Graviola & $\mathrm{X}$ & $\mathrm{X}$ & & $\begin{array}{l}\text { IAC } \\
42599\end{array}$ \\
\hline \multirow[t]{2}{*}{ Apiaceae } & Foeniculum vulgare Mill. & Erva-doce & $\mathrm{X}$ & & & IAC 48436 \\
\hline & $\begin{array}{l}\text { Petroselinum sativum } \\
\text { Hoffm. }\end{array}$ & Salsinha & $\mathrm{X}$ & & & I.C. \\
\hline Asparagaceae & $\begin{array}{l}\text { Herreria salsaparilha } \\
\text { Mart. }\end{array}$ & Salsaparrilha & & $\mathrm{X}$ & $\mathrm{X}$ & IAC 48826 \\
\hline \multirow[t]{7}{*}{ Asteraceae } & $\begin{array}{l}\text { Acanthospermum australe } \\
\text { (Loefl.) Kuntze }\end{array}$ & Carrapichinho & & & $\mathrm{X}$ & IAC 4334 \\
\hline & $\begin{array}{c}\text { Achyrocline satureioides } \\
\text { (Lam.) DC. }\end{array}$ & Macela-amarela & & & $\mathrm{X}$ & IAC 48437 \\
\hline & Ageratum conyzoides L. & Erva-de-são- João & & $\mathrm{X}$ & $\mathrm{X}$ & IAC 4545 \\
\hline & $\begin{array}{l}\text { Baccharis conyzoides } \\
\text { (Less.) DC. }\end{array}$ & Carqueja & & $\mathrm{X}$ & $\mathrm{X}$ & IAC 48440 \\
\hline & Bidens pilosa $\mathrm{L}$. & Picão & $\mathrm{X}$ & $\mathrm{X}$ & $\mathrm{X}$ & IAC 48438 \\
\hline & $\begin{array}{c}\text { Chamomilla recutita }(\mathrm{L} .) \\
\text { Rauschert }\end{array}$ & Camomila & $\mathrm{X}$ & & & I.C. \\
\hline & Mikania hirsutissima DC. & Cipó-cabeludo & & & $\mathrm{X}$ & IAC 48439 \\
\hline Bromeliaceae & Ananas comosus (L.) Merr. & Abacaxi & & & $\mathrm{X}$ & I.C. \\
\hline Chloranthaceae & $\begin{array}{c}\text { Hedyosmum brasiliense } \\
\text { Mart. ex Miq. }\end{array}$ & Bugre & & & $\mathrm{X}$ & IAC 48475 \\
\hline Commelinaceae & $\begin{array}{c}\text { Dichorisandra thyrsiflora } \\
\text { J.C. Mikan }\end{array}$ & Cana-da-índia & & & $\mathrm{X}$ & IAC 48442 \\
\hline Costaceae & Costus spicatus (Jacq.) Sw. & Cana-do-brejo & & & $\mathrm{X}$ & IAC 48443 \\
\hline Crassulaceae & $\begin{array}{l}\text { Kalanchoe crenata } \\
\text { (Andrews) Haw. }\end{array}$ & Saião & $X$ & $\mathrm{X}$ & & IAC 4150 \\
\hline Cucurbitaceae & Sicyos edulis Jacq. & Chuchu & $\mathrm{X}$ & & $\mathrm{X}$ & IAC 48476 \\
\hline Equisetaceae & Equisetum hyemale L. & Caninha-do-brejo & & & $\mathrm{X}$ & IAC 45333 \\
\hline Euphorbiaceae & Euphorbia prostrata Aiton & $\begin{array}{l}\text { Quebra-pedra- } \\
\text { verdadeiro }\end{array}$ & & & $\mathrm{X}$ & I.C. \\
\hline
\end{tabular}

\begin{tabular}{|c|c|c|c|c|}
\hline Fabaceae & Bauhinia forficata Link. & Unha-de-vaca & $\mathrm{X}$ & I.C. \\
\hline Geraniaceae & Geranium sp. & Malva-de-cheiro & $X$ & IAC 48489 \\
\hline
\end{tabular}




\begin{tabular}{|c|c|c|c|c|c|c|}
\hline Família & Espécies & Nome Popular & HPT & CLT & PRB & $\mathrm{N}^{0}$ exsicata \\
\hline \multirow{9}{*}{ Lamiaceae } & $\begin{array}{l}\text { Lavandula angustifolia } \\
\text { Mill. }\end{array}$ & Sálvia & $\mathrm{X}$ & & & IAC 18295 \\
\hline & Melissa officinalis L. & Melissa & $\mathrm{X}$ & & & I.C. \\
\hline & Mentha crispa L. & Hortelã-rasteiro & $\mathrm{X}$ & & & I.C. \\
\hline & Mentha spicata $\mathrm{L}$. & Hortelã-preta & $\mathrm{X}$ & & & IAC 4822 \\
\hline & Ocimum gratissimum $\mathrm{L}$. & Alfavaca-cravo & $\mathrm{X}$ & & & IAC 48445 \\
\hline & $\begin{array}{c}\text { Ocimum carnosum } \\
\text { (Spreng.) Link \& Otto ex } \\
\text { Benth. }\end{array}$ & Atroveram & $\mathrm{X}$ & & $\mathrm{X}$ & IAC 44394 \\
\hline & $\begin{array}{c}\text { Hyptis radicans } \\
\text { (Pohl) Harley \& J.F.B. } \\
\text { Pastore }\end{array}$ & Hortelã-de-bugre & & & $\mathrm{X}$ & IAC 49728 \\
\hline & Rosmarinus officinalis L. & Alecrim & $\mathrm{X}$ & & & IAC 48446 \\
\hline & Salvia officinalis L. & Sálvia & $\mathrm{X}$ & & & I.C. \\
\hline Lauraceae & Persea americana Mill. & Abacate & & & $\mathrm{X}$ & I.C. \\
\hline Lythraceae & $\begin{array}{c}\text { Cuphea carthagenensis } \\
\text { (Jacq.) J.F.Macbr. }\end{array}$ & Sete-sangrias & $\mathrm{X}$ & $\mathrm{X}$ & & IAC 7167 \\
\hline Malpighiaceae & Malpighia glabra L. & Acerola & & $\mathrm{X}$ & & IAC 48444 \\
\hline Moraceae & Morus nigra L. & Amora & $\mathrm{X}$ & & $\mathrm{X}$ & IAC 48477 \\
\hline \multirow[b]{2}{*}{ Myrtaceae } & Eugenia uniflora L. & Pitanga & $\mathrm{X}$ & & $\mathrm{X}$ & IAC 48447 \\
\hline & $\begin{array}{c}\text { Syzygium cumini (L.) } \\
\text { Skeels }\end{array}$ & Maria-preta & & & $\mathrm{X}$ & I.C. \\
\hline Oxalidaceae & Averrhoa carambola $\mathrm{L}$. & Carambola & & $\mathrm{X}$ & $\mathrm{X}$ & IAC 48448 \\
\hline Passifloraceae & Passiflora edulis Sims & Maracujá & $\mathrm{X}$ & & & IAC 48449 \\
\hline Piperaceae & Piper umbellatum L. & Capeva & & & $\mathrm{X}$ & IAC 48450 \\
\hline Phyllanthaceae & Phyllanthus tenellus Roxb. & $\begin{array}{l}\text { Quebra-pedra- } \\
\text { falso } \\
\end{array}$ & & & $\mathrm{X}$ & IAC 48444 \\
\hline \multirow{4}{*}{ Poaceae } & $\begin{array}{c}\text { Cymbopogon citratus (DC.) } \\
\text { Stapf }\end{array}$ & Cidreira & $\mathrm{X}$ & & & I.C. \\
\hline & Imperata brasiliensis Trin. & Sapê & & & $\mathrm{X}$ & I.C. \\
\hline & Saccharum officinarum L. & Cana-de-açúcar & $\mathrm{X}$ & $\mathrm{X}$ & & I.C. \\
\hline & Zea mays L. & Milho & & & $\mathrm{X}$ & I.C. \\
\hline Rosaceae & Pyrus communis $\mathrm{L}$. & Pera & & & $\mathrm{X}$ & I.C. \\
\hline \multirow{2}{*}{ Rutaceae } & Citrus sp. & Lima & $\mathrm{X}$ & $\mathrm{X}$ & & I.C. \\
\hline & Citrus sp. & Limão-rosa & & $\mathrm{X}$ & & I.C. \\
\hline \multirow{2}{*}{ Solanaceae } & Solanum cernuum Vell. & Panacéia & & & $\mathrm{X}$ & IAC 42852 \\
\hline & Solanum melongena L. & Berinjela & & $\mathrm{X}$ & & I.C. \\
\hline Trigoniaceae & Trigonia nivea Cambess. & Cipó-prata & & & $\mathrm{X}$ & $\begin{array}{c}\text { IAC } \\
48451\end{array}$ \\
\hline Tropaeolaceae & Tropaeolum majus L. & Cocolhar & $\mathrm{X}$ & & & IAC 48452 \\
\hline Urticaceae & Cecropia glaziovii Snethl. & Embaúva & $\mathrm{X}$ & & & IAC 48441 \\
\hline Verbenaceae & $\begin{array}{c}\text { Lippia alba (Mill.) N.E. Br. } \\
\text { ex P. Wilson }\end{array}$ & Erva-cidreira & $\mathrm{X}$ & & & IAC 48478 \\
\hline Vitaceae & $\begin{array}{l}\text { Cissus verticillata (L.) } \\
\text { Nicholson \&C.E. Jarvis }\end{array}$ & Uva-ursina & & & $\mathrm{X}$ & IAC 45096 \\
\hline
\end{tabular}

O sistema médico de classificação (doenças, sintomas, formas de prevenção e cura) dentro do grupo de entrevistados do Bairro dos Marins, como qualquer outro aspecto cultural desta comunidade, está constantemente se modificando e se adaptando como uma resposta ao universo dinâmico onde essa 
sociedade se insere. Nesta realidade, fatores culturais, sociais, ecológicos e influências inter e intraculturais não podem ser isolados e analisados separadamente. Para entender cada uma dessas interações é preciso um olhar amplo e minucioso por parte do pesquisador. De outro lado, vemos estudos sobre o conhecimento popular de plantas medicinais gerar produtos farmacêuticos com qualidades adequadas ao interesse de uma indústria milionária. Infelizmente esta realidade de desenvolvimento de novos produtos não tem promovido o bem-estar das comunidades estudadas, que se encontram ameaçadas e desprezadas tanto em seu "saber popular" quanto na matéria-prima (animais, plantas, microrganismos) onde está embasado este conhecimento. Com a abordagem êmica é possível verificar que para as três doenças propostas no estudo existem um sistema de classificação e diagnose popular próprio da comunidade, por vezes influenciada pela medicina moderna, em outras com base em experiências pessoais e coletivas, como a de se associar o tratamento do colesterol a plantas usadas há muitos anos na comunidade, para depurar e limpar o sangue.

\section{Características botânicas e ecológicas das plantas utilizadas}

As famílias botânicas de maior representatividade foram Lamiaceae 15\%, Asteraceae $12 \% \mathrm{e}$ Poaceae $7 \%$. Os hábitos das plantas de uso medicinal indicadas são predominantemente herbáceos terrícolas (47\%), seguidos de arbóreos (24\%), arbustivos (16\%) e trepadores herbáceos ou lianas (13\%). A preferência para o uso e o conhecimento de espécies herbáceas podem estar relacionados tanto a sua fácil disponibilidade de coleta e ao manejo deste tipo de planta quanto aos fatores relacionados à sua atividade biológica. Baseado na teoria da evidência e da disponibilidade, Stepp e Moerman (2001 apud GAZZANEO et al., 2003) sugerem que estas plantas concentram compostos biológicos muito ativos em função de seu hábito ou de suas estratégias de vida. Somando-se os principais locais de coleta das plantas (horta, quintal e pasto), $82 \%$ das plantas são coletadas em ambiente onde a grande maioria de plantas é herbácea terrícola, corroborando os dados de forma de vida (hábito) das plantas encontradas.

Para o preparo de remédios caseiros, verificou-se que a folha (51\%) é a parte vegetal mais utilizada, seguida de planta inteira (9\%) e folhas e frutos (9\%) (Figura 3).

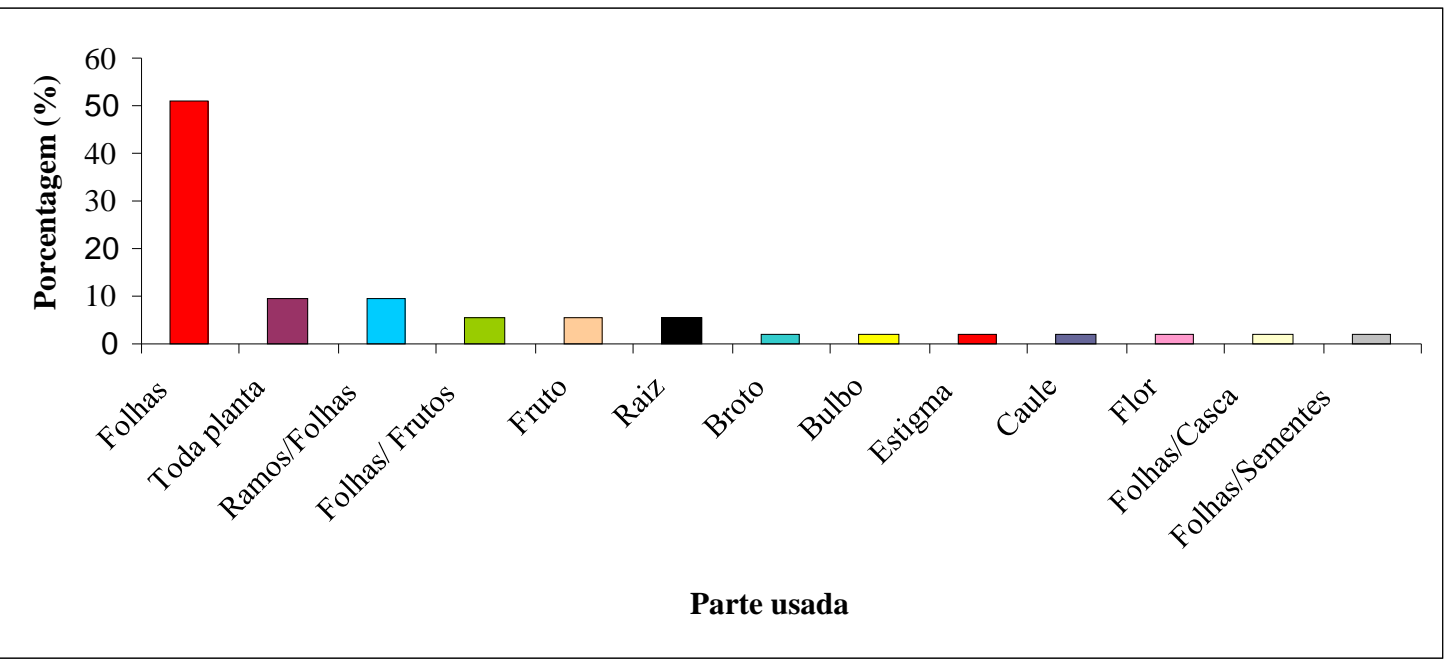

Figura 3: Parte vegetal das plantas utilizadas como medicinais pelos especialistas entrevistados para as doenças estudadas no Bairro dos Marins, Piquete- SP.

Segundo Hidalgo (2003), a folha é o órgão vegetal onde ocorre a fotossíntese, processo pelo qual produz açúcares e outros produtos biologicamente ativos, que serão metabolizados e usados para o crescimento da planta e demais processos essenciais para a vida do vegetal. Dentre as substâncias produzidas estão diversos compostos secundários, muitos dos quais usados na produção de fármacos ou como modelo para a síntese de moléculas. No Bairro dos Marins, as folhas são usadas frescas ou secas e o processo de secagem e armazenagem das partes dos vegetais varia de acordo com o entrevistado e com a espécie usada; porém, observou-se que $95 \%$ dos entrevistados têm o costume de usar as folhas ainda frescas no preparo dos remédios caseiros. Esses dados também são verificados nos trabalhos de Liporacci e Simão (2013) e Miranda et al. (2016) realizados com moradores de áreas urbano/rurais. Os principais modos de preparo dos remédios caseiros são apresentados na Figura 4. 


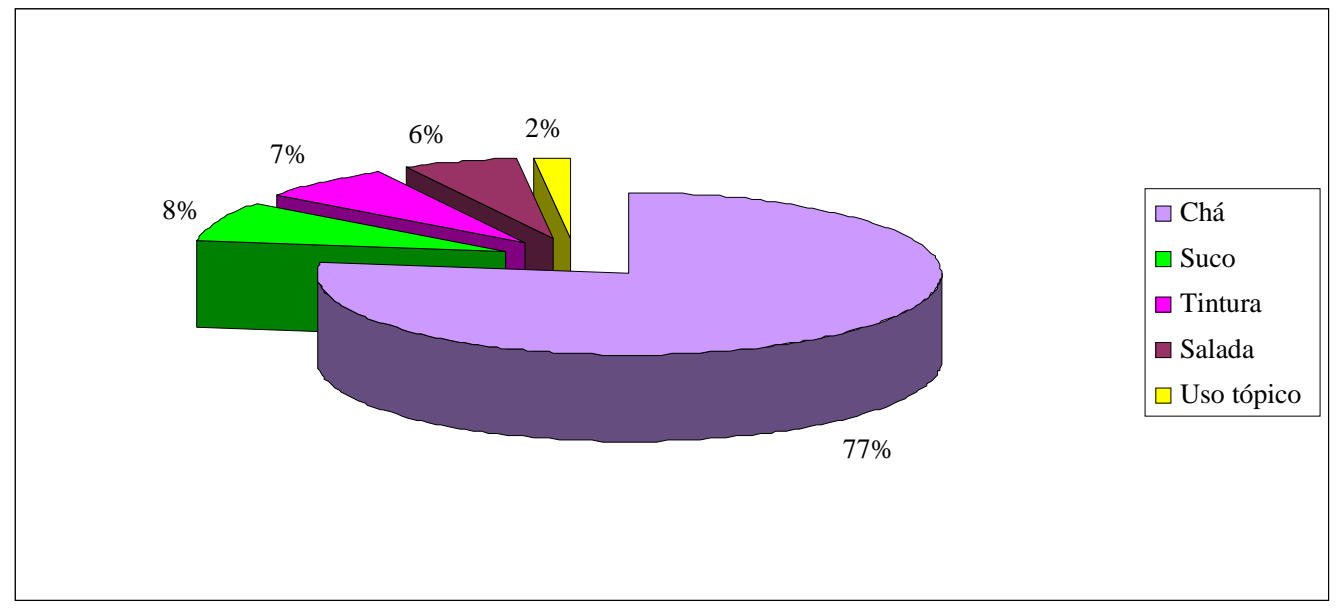

Figura 4: Formas de utilização das plantas medicinais citadas pelos especialistas entrevistados no Bairro dos Marins, Piquete- SP.

Kubo (1997), Costa (2002) e Santos (2006), estudando plantas medicinais em comunidades rurais, observaram que a forma de preparo mais utilizada pelos moradores locais é o chá. O mesmo foi observado no Bairro dos Marins onde, entre os entrevistados, há uma tendência a chamar de chá todas as formas de preparo onde a planta passa por um processo de fervura, mesmo que isso ocorra despejando a água quente em cima da planta, e não necessariamente fervendo ou cozinhando a planta na água. Quanto aos critérios usados na escolha do momento de coleta das plantas medicinais, $100 \%$ dos entrevistados responderam que o principal critério é a aparência da planta, ou seja, que os espécimes estejam sadios. Quanto a outros critérios citados para o momento da coleta, reportam-se às fases que a Lua se encontra (35\%) e à hora do dia $(25 \%)$. De acordo com os entrevistados, algumas espécies citadas na pesquisa, para que tenham o efeito esperado, devem ser coletadas ou no final da tarde ou no início da manhã. Os entrevistados relataram que a melhor hora do dia para se coletar as plantas medicinais é no início da manhã.

\section{CONCLUSÃO}

Os resultados encontrados reforçam o pensamento de que a Serra da Mantiqueira é berço de um rico conhecimento tradicional e de importantes recursos naturais da Mata Atlântica. E que a comunidade estudada apresenta vínculo estreito com a região da Serra da Mantiqueira, tanto no aspecto sociocultural quanto econômico.

Embora se tenha restringido a alguns grupos de doenças, com o levantamento tornou-se possível verificar que no Bairro dos Marins existe uso intenso de plantas medicinais nos cuidados primários à saúde, para os grupos das doenças estudadas, e que a forma de lidar com as doenças "modernas", onde os sintomas e os tratamentos precisam ser diagnosticados por laboratórios e por médicos, estão sendo, por essa comunidade, traduzidos e inseridos dentro da rica cultura tradicional. Essa tradução é feita no dia a dia em conversas com vizinhos, padres que trabalham nas pastorais da saúde, médicos que atendem no Bairro, programas de televisão e rádio, ouvidos e assistidos por eles e, principalmente, nas relações com familiares, mostrando que o conhecimento popular é dinâmico e se modifica de acordo com o tempo e espaço onde a comunidade se insere.

\section{AGRADECIMENTOS}

Os autores agradecem aos seguintes botânicos pelas identificações das plantas coletadas: Prof. Dr. Lin Chau Ming, Faculdade de Ciências Agronômicas da Unesp, de Botucatu-SP, Dra. Inês Machiline Silva, Dra. Elsie Franklin Guimarães, do Jardim Botânico do Rio de Janeiro, e da Dra Roseli Buzanelli Torres, pesquisadora do Núcleo de Pesquisa e Desenvolvimento do Jardim Botânico e curadora do herbário do Instituto Agronômico de Campinas (IAC). 


\section{REFERÊNCIAS}

ALEXIADES, M.N. Selected guidelines for ethnobotanical research: a field manual. The New York Botanical Garden, Bronx, New York, 306p. 1996.

AMOROZO, M.C.M. A abordagem etnobotânica na pesquisa de plantas medicinais. In: DI STASI, L. C. (Org.). Plantas medicinais: arte e ciência. São Paulo: UNESP, 1996. p. 47-68.

APG IV. Angiosperm Phylogeny Group IV. An update of the angiosperm phylogeny group classification for the orders and families of lowering plants: APG IV. Bot. J. Linn. Soc., 2016, p. 1-20.

BERNARD, H.R. Research methods in cultural anthropology. Newbury Park: SAGE, 1988. 520p.

CARRARA. D. Possangaba: pensamento médico popular. Editora Global, Rio de Janeiro. 1995.

COSTA, M.A.G. Aspectos etnobotânicos do trabalho com plantas medicinais realizado por curandeiros no município de Iporanga, SP. 2002, 134 f. Dissertação de Mestrado (Mestrado em Ciências Agronômicas/Horticultura) - Faculdade de Ciências Agronômicas, Universidade Estadual Paulista. Botucatu, 2002.)

FLORA DO BRASIL 2020 em construção. Jardim Botânico do Rio de Janeiro. Disponível em: < http://floradobrasil.jbrj.gov.br/ >. Acesso em: 08 Nov. 2019

GAZZANEO, L.R, LUCENA, R.R.P. AND ALBUQUERQUE, U.P. Knowledge and use of medicinal plants by local specialists in an region of Atlantic Forest in the state of Pernambuco (Northeastern Brazil). Journal of Ethnobiology and Ethnomedicine, v.1, n. 9, p. 1-9. 2005.

HENTGES, A.M. Dados socioculturais de usuários de plantas medicinais e plantas utilizadas como medicinais no município de Cândido Godói, Rio Grande do Sul. (Monografia). Licenciatura em Ciências Biológicas, Universidade da Fronteira Sul, Cerro Largo, RS, 2015, 55p.

HIDALGO, A.F. Plantas de uso popular para o tratamento da malária e males associados da área de influência do Rio Solimões e Região de Manaus, AM. Tese (Doutorado em Horticultura) - Universidade Estadual Paulista, Botucatu, SP. 2003

KUBO, R.R. Levantamento das plantas de uso medicinal em Coronel Bianco, RS. Porto Alegre, RS,

1997. 161 f. Dissertação (Mestrado em Botânica) - Universidade Federal do Rio Grande do Sul, RS.

LIPORACCI, H.S.N.; SIMÃO, D.G. Levantamento etnobotânico de plantas medicinais nos quintais do

Bairro Novo Horizonte, Ituiutaba, MG. Rev. Bras. Pl. Med., Campinas, v.15, n.4, p.529-540, 2013.

MARTIN, G.J. Ethnobotany. Chapman \& Hall. London.UK, 268p. 1995.

MATOS, F.J.A. Guia fitoterápico. 2 ed. Editora Universidade Federal do Ceará, Fortaleza. 340p. 2001.

MEDEIROS, P.M.; FERREIRA-JÚNIOR, W.S.; RAMOS, M.A.; SILVA, T.C.da, LADIO, A.H.; ALBUQUERQUE, U.P. Why do people use exotic plants in their local medical systems? A systematic review based on Brazilian local communities. PLOS ONE, v. 6, September 27, P.1-14, 2017

MIRANDA, T.G.; OLIVEIRA-JÚNIOR, J.F.; MARTINS-JÚNIOR, A da S, TAVARES-MARTINS, A.C.C. O uso de plantas em quintais urbanos no bairro da Francilândia no município de Abaetetuba, Pará, Brasil, Scientia Plena, v.12, n.06, p.1-18, 2016.

MYERS, N; MITTERMELER, A. R.; MITTERMELER, C. G.; FONSECA, G.B.A.; KENT, J. 2000. Biodiversity Hotspots for conservation priorities. Nature 403: 853-858.

SANTOS, J.F.L. Uso popular de plantas medicinais na comunidade rural da Vargem Grande, Município de Natividade da Serra, SP. 2006, 104 f. Dissertação de Mestrado (Mestrado em Ciências Agronômicas/Horticultura) - Faculdade de Ciências Agronômicas, Universidade Estadual Paulista. Botucatu, 2006.

PIZZIOLO,V.R.; BRASILEIRO, B.G.;OLIVEIRA, T.T.1 ; NAGEM, T.J. Plantas com possível atividade hipolipidêmica: uma revisão bibliográfica de livros editados no Brasil entre 1998 e 2008. Rev. Bras. Pl. Med., Botucatu, v.13, n.1, p.98-109, 2011

STANISKI, A.; FLORIANI, N.; STRACHULSKI, J. Estudo etnobotânico de plantas medicinais na comunidade faxinalense Sete Saltos de Baixo, Ponta Grossa - PR, Terr@Plural, Ponta Grossa, v.8, n.2, p. 321340, jul/dez. 2014. 